\title{
Resonant and Non-resonant Amplification of Massless Gauge Fields during an Oscillating Dilaton Phase
}

\author{
Massimo Giovannini证 \\ DAMTP, Silver Street, Cambridge, CB3 9EW, United Kingdom
}

\begin{abstract}
The resonant and non-resonant amplification of electromagnetic inhomogeneities is investigated within a phase of coherent dilaton oscillations. We show, in some explicit toy model, how the critical energy density bound applied to the amplified fluctuations of the massless gauge fields can constrain the dilaton mass.
\end{abstract}

Accepted for publication in Physical Review D

\footnotetext{
${ }^{1}$ e-mail: M.Giovannini@damtp.cam.ac.uk
} 


\section{Introduction}

The coherent oscillations of a massive scalar field minimally coupled to gravity are a possible source of many cosmological problems which can result in a quite stringent set of bounds on the value of the scalar mass. The implications of the so-called "Polonyi potential" were analysed in different contexts [1, 2, 3, 国.

The energy density stored in the coherent oscillations red-shifts faster than the surrounding relativistic plasma and its contribution might become soon quite significant depending upon the initial amplitude of the scalar field at the beginning of the oscillating phase. If the initial amplitude is of the order of the Planck mass (as it seems natural to require for interactions of gravitational strength) the red-shifted energy density of the oscillating field might have catastrophic consequences for the big-bang nucleosynthesis.

At the nucleosynthesis curvature scale $H_{N}\left(t_{N}\right)$ the background temperature should be $T_{N} \sim O(1 M e v)$, implying a bound for the reheating temperature associated with the scalar field decay and a lower bound for the scalar field mass which should be heavier than $10^{4} G e v$. The decay of the coherent oscillations might have dangerous consequences even before the nucleosynthesis scale because baryogenesis forbids a too high entropy release during the decay of the scalar field $\left(\Delta S \lesssim 10^{5}\right)$ in order not to wash-out any pre-existing baryon asymmetry [5], turning, ultimately, into a further lower bound on the mass which should be larger than $10^{14}$ Gev. A possible solution to the Polonyi problem could seem to fine-tune the initial amplitude of the classical background oscillations to be much smaller than 1 (in Planck units).

In this case on top of the constraints on the classical scalar background one should also be aware of other possible bounds coming from the scalar field fluctuations whose nonrelativistic modes might be amplified, for instance, during some inflationary epoch preceeding the coherent oscillations regime [2].

String moduli are coupled to Standard Model fields only through Planck scale suppressed interactions and their effective potential is exactly flat at small coupling in the supersymmetric limit, but can become curved thanks to non-perturbative effects or because supersymmetry breaking. String moduli, then, also suffer of the Polonyi problem [5, 7]. Among the 
moduli fields a very special role is played by the dilaton background which provides a unified value for the gravitational and gauge coupling constant at the String scale $\lambda_{S}$ and which then couples not only to the Einstein-Hilbert term but also, for example, to the abelian and non-abelian gauge fields [8] present in the theory. Precision tests on the equivalence principle would seem to imply a lower bound for the dilaton mass $m[9]\left(m>10^{-4} \mathrm{ev}\right)$ and, unless we do adopt the approach of [10] (where a very light dilaton mass is postulated), the problem of the coherent oscillations persists.

One possibility would be to fine-tune the initial amplitude of the dilaton to be much smaller than one (in Planck units) and then the possible constraints coming from the amplified dilaton fluctuations seem quite alleviated provided they are amplified with spectrum increasing in frequency [11] as it seems reasonable in a string cosmological scenario [12]. There have been recently few interesting suggestions in order to solve the moduli problem - A second stage of inflation able to dilute the energy of the moduli field was suggested [13 and, more recently, it was also pointed out that a mass term contribution of the order $C m^{2} H^{2}(t)$ (with $C>>1$ ) could smear the amplitude of the initial coherent oscillations definitely relaxing the problem [5].

Our purpose in this paper is not so ambitious and we will not specifically address the moduli problem. We want only to point out that the amplification of the gauge fields excited by the coherent oscillations of the dilaton coupling should also be taken into account in the same way as the scalar field fluctuations were shown [2] to be important in the standard Polonyi problem. It is actually well known that in General Relativity the only fields which can be amplified through the evolution of time-dependent background geometry are the ones whose equations of motion are not invariant under the Weyl rescaling of the metric tensor (such as, for example, scalar and tensor inhomogeneities [14]) and since the evolution equations of the Maxwell fields (and of the chiral fermions) are invariant under the conformal rescaling of the metric background, it was also correctly concluded that neither the electromagnetic inhomogeneities nor the chiral fermions can be successfully amplified. In string inspired backgrounds the situation changes and gauge fields fluctuations might grow thanks to the dilaton coupling in the same way as the coupling to the background curvature allows the parametric amplification of gravity waves in General Relativity. 
The amplification of the electromagnetic fluctuations was discussed in the context of the magnetic field generation [15], but not studied, so far, in the context of the coherent oscillations of the dilaton field.

In the low energy string theory effective action [8] the evolution equations describing the dynamics of the massless modes can be written either in the Einstein frame (where the Einstein-Hilbert term and the dilaton field are formally decoupled and the Planck scale is really a constant) or in the String frame (where the coupling of the dilaton to the Einstein Hilbert term is explicit and the typical scale of the theory is the String scale [while the Planck scale is no longer a constants since it evolves in time as $\left.\left.\lambda_{P}=e^{\frac{\phi}{2}} \lambda_{S}\right]\right)$. As long as the corrections in the string tension are not included the two frames are equivalent (up to a conformal transformation and to a ri-definition of the dilaton field). Moreover it was checked that the amplitude of the excited inhomogeneities is exactly the same in both the description even though the treatment in the String frame can be, in principle, more cumbersome [24]. Since we are mainly concerned, in the present discussion, with small curvature scales $(H<$ $\left.M_{P}\right)$ and small dilaton couplings $\left(e^{\phi / 2} \ll 1\right)$ we find useful to work, from the very beginning, in the Einstein frame, where the low energy effective action takes the form:

$$
S=-\int d^{4} x \sqrt{-g}\left(R-\frac{1}{2} g^{\mu \nu} \partial_{\mu} \phi \partial_{\nu} \phi+V(\phi)+\frac{1}{4} e^{-\phi} F_{\mu \nu}^{a} F^{a \mu \nu}+\ldots\right)
$$

(we adopted "natural" units by setting $16 \pi G=1$ and we neglected the possible contribution of the antisymmetric tensor field which is not directly relevant for the problem which we are discussing; $F_{\mu \nu}^{a}$ is a generic gauge field which will be considered abelian; $\phi=\Phi-\ln V_{6}=\ln g^{2}$ is connected with the four dimensional gauge coupling $[\Phi$ is the 10-dimensional dilaton field and $V_{6}$ is the volume of the 6-dimensional compact internal space] ).

The origin of the dilaton potential $V(\phi)$ is non perturbative and related to supersymmetry breaking. On theoretical ground, in critical superstring theory the dilaton potential has to go zero as a double exponential as $\phi \rightarrow-\infty$ (weak coupling), namely $V(\phi) \sim \exp \left(-c^{2} \exp (-\phi)\right)$ with $c^{2}$ a positive (model dependent) constant. On more physical ground $V(\phi)$ is believed to have also one (or more) minima for some finite value of the dilaton $\phi \sim \phi_{\text {min }}$. In general we can say that after a phase of decreasing coupling driven either by the dilaton's kinetic energy or by a radiation dominated background the coherent oscillations start at a curvature scale $H \sim m$ [11, [1]. 
The fluctuations of the gauge fields can then be amplified in two different ways : either through the oscillating phase itself (for Fourier modes $k \lesssim m$ ) or through the sudden change from the (model-dependent) pre-oscillating epoch to the oscillating one. In the first case the gauge fields are amplified in a resonant way with a mechanism very similar to the ones recently introduced in the studies of the so-called explosive inflaton decay [16, 17] during the pre-heating dynamics [16, 18, 19, 20]. In the second case the gauge fields are amplified in a non-resonant way since the main effect will be given not by the oscillations themselves but by the the change of the background evolution towards a phase effectively dominated by a non-relativistic pressure-less fluid which ends up, ultimately, with the final dilaton decay at a curvature scale $H_{d} \sim \Gamma \sim m^{3} / M_{P}^{2}$. The amplified electromagnetic inhomogeneities should not over-close the universe and then the energy density of the fluctuations should be always smaller than the energy density of the relativistic plasma. The critical energy density bound applied on the amplified fluctuations can then be translated into a bound on the parameters of the background during the phase of the coherent oscillations, leading to possible bounds on the dilaton mass.

The outlined picture of the dilaton relaxation might be unreliable for many reasons. By studying the coherent oscillations only in the vicinity of the minimum of the potential we will tacitely assume that the potential is only quadratic. Detailed studies of the preheating dynamics both in flat 20] and curved space [21] showed that this approximation is quite misleading. The quadratic potential is in fact, by itself, unstable under renormalization and a $\lambda \phi^{4}$ is needed from the one-loop divergences. In the case of the present paper, moreover, even higher powers are expected given the double exponential form of $V(\phi)$. Our results will not have the same degree of accuracy of Ref. [20, 21] also because we do not include the back-reaction effect of the produced fluctuations on the dynamics of the background as carefully done in [21] in the case of de Sitter space.

The case which we are going to discuss is also quite peculiar because of the exponential coupling of the dilaton to the gauge fields which was not specifically considered in any of the previous discussions of the preheating dynamics. This limitations will be reflected in our calculations and we will come back to this point later.

The plan of this paper is then the following. Sec. II is devoted to the study of the resonant 
amplification of electromagnetic fluctuations occurring for modes $k \gtrsim m$, while in Sec. III we will turn our attention to the non-resonant case $(k \lesssim m)$ and we will show, within some very elementary example, how the bounds on the amplified electromagnetic fluctuations can be turned into bounds on the dilaton decay scale and then on the dilaton mass.

\section{Resonant Amplification}

In a conformally flat metric of FRW type

$$
g_{\mu \nu}=\operatorname{diag}\left(1,-a^{2}(t),-a^{2}(t),-a^{2}(t)\right)
$$

the background equations derived from the action (1.1) become

$$
\begin{aligned}
H^{2} & =\frac{1}{12}\left(\dot{\phi}^{2}+2 V\right), \quad \dot{H}=-\frac{1}{4} \dot{\phi}^{2} \\
\ddot{\phi} & +3 H \dot{\phi}+\frac{\partial V}{\partial \phi}=0
\end{aligned}
$$

$\left(H=(\ln a)^{\cdot},{ }^{\prime}=\partial / \partial t\right)$. For scales $H \lesssim H_{\max }<M_{P}$ ( where $H_{\max }$ is the maximal curvature scale during the pre-oscillating phase ) the evolution of the dilaton can be quite different depending upon the particular model (see for instance [7, 11, 12]). Our discussion does not rely on any special background during the pre-oscillating phase but only on the existence of such a phase, occurring for $H>m$. We will only assume that the curvature scale and the coupling constant $\left(g=e^{\frac{\phi}{2}}\right)$ are both decreasing in time $(\dot{H}<0, \dot{g}<0)$.

For example in the post-big-bang evolution of the String cosmological models the background (right after $\left.H_{\max }\right)$ is dominated by radiation $(H \simeq 1 / 2 t)$ and the dilaton decreases

according to the third equation in (2.2) reaching a constant value before the onset of the coherently oscillating regime, i. e. $\phi=\phi_{0}+\phi_{1}\left(H / H_{\max }\right)^{1 / 2}$. If the background is dominated, during the pre-oscillating phase, by the dilaton's kinetic energy [7, 12] $\left(H \sim 1 / 3 t, \rho_{\phi} \sim a^{-6}\right)$ the dilaton decreases as $\phi=\phi_{0}+\phi_{1} \ln \left(H_{\max } / H\right)$. The potential appearing in Eq. (2.2) can be approximated (according to the considerations of the Introduction) by [22]

$$
V(\phi)=\frac{m^{2}}{2}\left(\phi-\phi_{\text {min }}\right)^{2} \exp \left(-c^{2} \exp (-\phi)\right) .
$$

In the following discussion we will keep only the quadratic terms in the potential. This approximation will allow our estimates but at the same time we stress again that already 
the (flat space) solutions of the theory including quartic self-interactions might turn out qualitatetively and quantitatively different giving rise to sizeable errors (up to 40\%) in the number of produced particles [23].

As soon as $H \sim O(m)$ the dilaton starts oscillating with initial amplitude $\phi_{0} \simeq O(1)$ in Planck units. Moreover, for interactions of gravitational strength we also expect $\phi_{\min } \simeq O(1)$ and, without fine-tuning, $\left|\phi_{0}-\phi_{\min }\right| \simeq O(1)$.

In the oscillating regime a self-consistent solution of the Eq. (2.2) with the potential $(2.3)$ is :

$$
\begin{gathered}
\phi(t)=\phi_{\text {min }}+\frac{4}{\sqrt{3}}\left(\frac{\phi_{0}^{+} \cos m t+\phi_{0}^{-} \sin m t}{m t}+\frac{\phi_{0}^{+} \sin m t \cos ^{2} m t+\phi_{0}^{-} \cos m t \sin ^{2} m t}{(m t)^{2}}\right)+O\left(\left(\frac{H}{m}\right)^{3}\right) \\
(2.4) \\
a(t)=a_{0}(m t)^{2 / 3}\left[1+\frac{\cos m t}{6(m t)^{2}}-\frac{1}{24(m t)^{2}}+O\left(\left(\frac{H}{m}\right)^{3}\right)\right]
\end{gathered}
$$

$\left(\right.$ with $\left.\left|\phi_{0}^{+}\right|^{2}+\left|\phi_{0}^{-}\right|^{2}=a_{0}^{2}\right)$.

The leading term (in $H / m$ ) of Eq. (2.5) turns out to be more important in the case of the non-resonant amplification which rely on the change in the background evolution among the pre-oscillating and the oscillating phase. It is easy to transform the previous expressions to conformal time $\eta(a(\eta) d \eta=d t)$ by keeping only the leading order behaviour in $H / m$ in Eq. (2.5). We will use the conformal time in order to describe the non-resonant amplification (where the expansion of the universe is mainly concerned). On the other hand it is far more convenient to analyse the resonant amplification in cosmic time where the solutions of the background equations can be directly inserted into the gauge fields evolution equations, leading, finally, to more tractable expressions.

From now on we will specialise our discussion to the case of abelian fields ( but our discussion can be easily extended to the non-abelian case provided $e^{\phi / 2} \ll 1$ ). The evolution equation for the Fourier modes of the abelian gauge fields (derived from the action (1.1) and in the gauge $\left.A_{0}=0, \nabla_{i} A^{i}=0\right)$ are, for each of the two physical polarizations

$$
\mathcal{A}_{i}(k)^{\prime \prime}+\left[k^{2}-g\left(g^{-1}\right)^{\prime \prime}\right] \mathcal{A}_{i}(k)=0, \quad g(\phi)=e^{\frac{\phi}{2}}
$$

(where $\mathcal{A}_{i}=g(\phi) A_{i}$ and ' $\equiv \partial / \partial \eta$ ). It is useful to rewrite the same equation in cosmic time

$$
\ddot{f}_{i}(\omega)+\left[\omega^{2}-G(t)\right] f_{i}(\omega)=0
$$


where $\omega=k / a, f_{i}=\sqrt{a} \mathcal{A}_{i}$ and

$$
G(t)=\left(\frac{g}{\sqrt{a}}\right)\left(\frac{\sqrt{a}}{g}\right)^{\cdot}=\frac{7}{48} \dot{\phi}^{2}-\frac{1}{2} \ddot{\phi}+\frac{1}{6} \frac{\partial V}{\partial \phi}+\frac{V}{24}
$$

(the second exact equality follows directly by repeated use of the background equations (2.2)). By inserting Eq. (2.4) in Eq. (2.8) and by keeping only the leading terms in $H / m$ we obtain that Eq. (2.7) can be rewritten as

$$
\frac{d^{2} f_{i}}{d z^{2}}+\left[\delta_{e f f}(z)-2 \epsilon_{e f f} \cos 2 z\right] f_{i}=0, \quad z=\frac{m t}{2}
$$

where $\delta_{\text {eff }}=4\left(\frac{k}{m}\right)^{2}(z / 2)^{-4 / 3}, \epsilon_{\text {eff }}=\epsilon z^{-1}\left(\epsilon=8 /(3 \sqrt{3}) \phi_{0}^{+}\right.$; in order to write the previous equation we have chosen, for simplicity, that $\phi_{0}^{-}=0$ in Eq. (2.4) and we also assumed $\phi_{\text {min }} \simeq \phi_{0}^{+}$). If we focus our attention on the regime where $k>>m$ (i. e. $H>>m$ ) we could neglect, in the first approximation the universe expansion and then $\delta_{\text {eff }} \simeq \delta=4(k / m)^{2} 2^{4 / 3}$, $\epsilon_{e f f} \simeq \epsilon$. In this resonant approximation Eq. (2.9) becomes exactly the well known Mathieu equation [26]. Notice that when $\epsilon=0$ and $\delta=2 n+1(n=0,1,2 \ldots)$ there is an exact solution of Eq. (2.9) with period $2 \pi$. If $\delta=2 n$ there is an exact solution with period $\pi$. We can then say that the instability boundaries in the $\delta-\epsilon$ plane will cross $\epsilon=0$ at the points $\delta=(2 n)^{2}$ and $\delta(2 n+1)^{2}$ which are the conditions for the parametric resonance to hold. This analysis can be pursued very easily if $\epsilon \ll 1$ (i.e. $\phi_{0}^{+} \ll 1$ ).

A more precise map of the boundaries of the unstable region can be obtained in the $\delta-\epsilon$ plane to the desired order in $\epsilon$. The analytic treatment shows that in this case the solutions of Eq. (2.9) are exponentially unstable but only in narrow resonance bands (see, for instance, [17, 19] and [25]) $\left|E_{n}\right| \simeq n^{2 n} 2^{3-2 n}[(n-1) !]^{-1} \epsilon^{n} \delta^{1-n}$ with $\delta=n \mp \frac{E_{n}}{2}$ and $E_{n}<\delta$. Each resonance band with $n>1$ corresponds to keeping terms $O\left(\epsilon^{n}\right)$ and then the width of each successive resonance shrinks but near the center of each resonance band the solutions take the form $f_{i}^{(n)}(z)=(4 \delta(k))^{-1 / 4} \exp \left( \pm \alpha_{k}^{(n)} z\right)$ (with $\alpha_{k}^{(n)}$ real and positive).

As it was extensively noticed in the context of the studies of the preheating dynamics in the narrow resonance regime the amplification of the inhomogeneities can only be very mild. Since in our case $\epsilon=8(3 \sqrt{3})^{-1} \sim 1.53$ we might argue that, provided we do not fine-tune $\phi_{0}^{+} \ll 1$ we are not in the narrow resonance regime but in the broad resonance. We notice that the mode function $G(t)$ appearing in Eq. (2.9) reduced simply to a damped cosinus only because we kept only quadratic terms in the dilaton potential in the vicinity of its minimum. 
As shown in [20] already the inclusion of $\lambda \phi^{4}$ term (required by stability under renormalisation) leads to a Lame' equation where the cosinus in the mode function is replaced by an elliptic cosinus. Needless to say that the most accurate analysis is performed using the Lame' equation. In the present case, however, the mode function would look even more complicated than the one of the usual Lame' equation (even if we keep only quartic selfinteractions in $V(\phi))$, because of the exponential coupling of the dilaton to the gauge fields which is very different from the usual couplings discussed in the context of the preheating dynamics [16, 17, 20].

The previous (and following) estimates based on Eq. (2.9) are then expected to be drastically changed in a fully consistent non-linear calculation and we only use it as a naive toy model but, none the less we find useful to illustrate with simple examples the effect we are discussing.

If in the case $\epsilon \sim 1$ we can have a resonance (in flat space) this can be expected to be shut off pretty soon by the universe expansion. A simple Runge-Kutta method can be employed after the second order differential equation (2.9) has been reduced to a system of two first order in the variables $Q \equiv f_{i}$ and $P \equiv f_{i}^{\prime}$. We took $\phi_{0}^{+}=1$ and $\epsilon=8(3 \sqrt{3})^{-1}$ and we explored different values of $k / m$ after having normalised $Q$ at the value of the quantum mechanical fluctuations $\left(Q\left(z_{0}\right)=f\left(z_{0}\right)=\left(4 \delta_{e f f}\left(z_{0}\right)\right)^{-1 / 4}\right)$, and $z_{0}=2$ [i.e. $t_{0}=1 / m$ ] is the initial integration time). The results illustrated in Fig. 1 emphasize the importance of the universe expansion. We plot $f_{i}(z)$ for different values of the parameters $k / m$ and $A=\phi_{0}^{+}$.

The growth of the solutions in flat space and the consequent particle production is seriously affected by the universe expansion. The solutions discussed in this section neglected completely the problem of the back-reaction of the produced particles on the evolution of the geometry. It was actually stressed in [16, 23] that the effect of the produced inhomogeneities modifies the effective equation of state from $p=0$ (in the regime of coherent oscillations) to $p=\rho / 3$ (when the produced fluctuations dominate). In our case we can estimate that the back-reaction effect can be safely neglected for times $\Delta t \sim 1 / m(\Lambda+O(H / m))$ where $\Lambda$ is a number which depends upon the particular model of dilaton relaxation and upon the amplitude of the dilaton (in Planck units) at the beginning of the oscilating phase. 

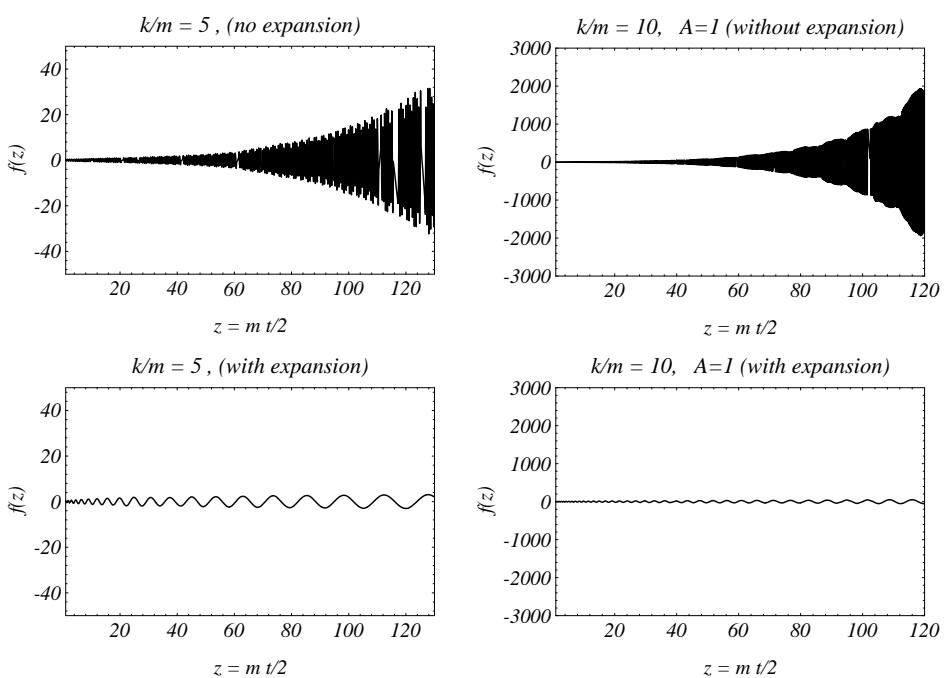

Figure 1: We plot few solutions of Eq. (2.11) for $A=\phi_{0}^{+}=1$ (i.e. $\epsilon=1.53$ ) in the resonant case $(k>m)$. The effect of the universe expansion on the flat space solutions is described.

\section{Non-resonant amplification}

We just showed that the amplification due to parametric resonance seems quite small and we now turn to the problem of non-resonant amplification occurring for modes $k \lesssim m$. We will discuss in this section a "minimal" toy model where the dilaton rolls down during a (model dependent) pre-oscillating phase, settles to its minimum and then decays at a curvature scale determined by its decay width $H_{d} \sim \Gamma \sim m^{3} / M_{P}^{2}$ producing a radiation dominated epoch. We are then led to study the amplification of massless gauge fields in the following model :

$$
g=e^{\phi / 2}=\left(\frac{\eta}{\eta_{\max }}\right)^{\beta}, \quad a(\eta) \sim\left(\frac{\eta}{\eta_{\max }}\right)^{\alpha}, \quad \eta<\eta_{d}, \quad \text { and } \quad a(\eta) \sim\left(\frac{\eta}{\eta_{d}}\right), \quad \eta>\eta_{d}
$$

(with $\beta<0$ and $\alpha<0$ ).

According to this simple model of background evolution the effective potential $V(\eta)=$ $g\left(g^{-1}\right)^{\prime \prime}$ appearing in Eq. (2.6) will be :

$$
V(\eta)=\frac{\beta(\beta+1)}{\eta^{2}}, \quad \eta<\eta_{d} \text { and } V(\eta)=0, \quad \eta>\eta_{d}
$$

A particular solution of Eq. (2.6) for $\mathcal{A}_{i}(k)$ can thus be written in terms of the first and second kind Hankel functions $H^{(1)}$ and $H^{(2)}$ corresponding to free oscillating modes in the $|\eta| \rightarrow \infty$ limit. 
The effective potential barrier leads to an amplification of the initial fluctuations, ore equivalently, to particle production from the vacuum. Starting with an incoming Fourier mode which is of positive frequency with respect to the asymptotic state at the left of the barrier $(\eta \rightarrow-\infty)$, one has in general, for $\eta \rightarrow \infty$ a linear combination of modes which are of positive and negative frequency with respect to the new vacuum. The mixing coefficients $c_{ \pm}$ define the Bogoliubov transformation connecting the two vacua and determine the spectral distribution of the amplified fluctuations.

The general solution of Eq. (2.6) in the two distinct epochs defined in Eq. (3.1)-(3.2) can be written for each Fourier mode $\mathcal{A}_{i}(k)$

$$
\mathcal{A}_{i}(k)=\frac{1}{\sqrt{k}} \sqrt{k \eta} H_{\mu}^{(2)}(k \eta), \quad \eta<\eta_{d}, \quad \text { and } \mathcal{A}_{i}(k)=\frac{1}{\sqrt{k}}\left(c_{+} e^{-i k \eta}+c_{-} e^{i k \eta}\right) \quad \eta>\eta_{d}
$$

The Bogoliubov coefficients $c_{ \pm}$can be fixed by the two conditions given by matching $\mathcal{A}_{i}$ and $\mathcal{A}_{i}^{\prime}$ in $\eta=\eta_{d}$. It is well known that the Bogoliubov coefficients determined in this "sudden" approximation can, in principle, lead to ultraviolet divergences in the energy density of the amplified fluctuations. The physical reason for this is that the modes with frequency $k>1 / \eta_{d}$ the sudden approximation cannot be rigourously applied and the calculation should be performed with a smooth effective potential barrier interpolating among the two vacua.

To smear the potential step will produce an exponential suppression of $\left|c_{-}\right|$for modes $k>1 / \eta_{d}$. Then in the frequency band we are interested in the amplification coefficient will be then given by $\left|c_{-}\right| \simeq\left(k \eta_{d}\right)^{-\frac{1}{2}-|\mu|}$ and the energy density of the produced massless gauge bosons $\rho$, per logarithmic interval of proper frequency $d \ln \omega$, is obtained by summing over the two polarisation states of the amplified waves:

$$
\frac{d \rho}{d \ln \omega}=2 N \omega^{4}\left|c_{-}\right|^{2}
$$

We included also the factor $N$ which counts the different massless gauge fields appearing originally in the low energy string theory effective action (1.1) which can be, in principle, also quite large, of the order of $10^{3}-10^{4}$ (as it happens, for example, in heterotic string theory [22]). In order to obtain the final expression of the spectral energy density of the amplified fluctuations in critical units we divide Eq. (3.4) by the radiation energy density 
produced by the dilaton decay $\left(\rho_{c}=H_{d}^{2} M_{P}^{2}\left(a_{d} / a\right)^{4}\right)$ and we obtain

$$
\Omega(\omega)=\frac{1}{\rho_{c}} \frac{d \rho}{d \ln \omega} \simeq N\left(\frac{\omega}{\omega_{d}}\right)^{3-2|\mu|}\left(\frac{H_{d}}{M_{P}}\right)^{2}
$$

The spectral energy density of the amplified inhomogeneities has to be always $\Omega(\omega, t)<1$ for all the frequencies and for all the times. If the energy spectrum is either flat or decreasing $(|\mu| \geq 3 / 2)$ the critical density bound should be applied at the lowest amplified frequency. If the spectrum is increasing $(|\mu|<3 / 2)$ the bound should be applied to the highest possible frequency which coincides, in our case with $\omega \sim \omega_{d}$. Therefore the critical density bound implies, respectively,

$$
\frac{m}{M_{P}} \lesssim N^{-\frac{1}{6}}\left(\frac{\omega_{i}}{\omega_{d}}\right)^{\frac{3-2|\mu|}{6}}, \text { for }|\mu| \geq \frac{3}{2} \quad \text { and, } \quad \frac{H_{d}}{M_{P}} \lesssim N^{-\frac{1}{2}} \text {, for }|\mu|<\frac{3}{2}
$$

where $\omega_{i}$ is the infrared scale of the spectrum. As an example we consider the case where during the pre-oscillating phase the evolution is driven by the kinetic energy of the dilaton starting from the scale $H_{\max }<M_{P}$. In this case $\beta=-\sqrt{3}$ and $|\mu|=(\sqrt{3}-1) / 2$. The critical density bound applied to the highest amplified frequency gives from (3.6) in terms of the dilaton mass $m \lesssim N^{-1 / 6} M_{P}$ (we used $H_{d} \simeq\left(m^{2} / M_{P}^{3}\right)$. It is amusing that the critical density bound implies a constraint for the dilaton mass which depends upon the number of the amplified massless gauge fields. If $N \simeq 2 \times 10^{3}$ this bound basically implies $m \lesssim 10^{-1} M_{P}$. Our example shows clearly that, in spite of the simplified model of background evolution it is possible to use the non-resonant amplification of massless gauge fields in order to constrain the dilaton mass. Since our purpose, in this section, is only illustrative, we only mention that it is possible to obtain more complicated exclusion plots either by leaving $\mu$ as a free parameter, or by adding to our "minimal" example the effect of the intermediate phase (occurring for $H_{d}<H<m$ ). We are anyway studying modes $k<m$ and the addition of this phase will only change the Bogoliubov coefficient $\left|c_{-}\right|$(and then the energy density of the amplified fluctuations) through the inclusion of an extra parameter (the duration of the intermediate phase) which will make broader (in conformal time units ) the "under-barrier" region in the effective potential appearing in Eq. (3.2). In these cases further constraints relating the dilaton decay scale to the other parameters of the background model can be obtained. 
We discussed the resonant and non-resonant amplification of massless gauge fields during a phase of dilaton oscillations and our conclusions are that the resonant amplification is a less efficient mechanism unless the value of the dilaton at the beginning of the oscillating phase is fine-tuned to be orders of magnitude bigger than the Planck mass which appears to be unreasonable for interactions of gravitational strength. The important caveat is, anyway, that the estimates we presented were done in some oversimplified model of dilaton relaxation and, for a more reliable answer, a complete non-linear analysis would be needed. The nonresonant amplification of massless gauge fields seem, already in our example, a more efficient mechanism and can very likely lead to possible constraints on the value of dilaton decay scale and on the value of the dilaton mass.

\section{Aknowledgments}

I am indebted to A. C. Davis and N. Turok for their interest in topics related to the present investigation and for the enjoyable atmosphere of DAMTP where part of the work was completed. I would like to thank Andrei Linde for useful discussions which partially motivated this investigation and N. Sanchez for the stimulating environment created at the Erice School of Astrophundamental topics where those discussions took place. I am very grateful to M. Shaposhnikov for many important insights on the various roles of amplified electromagnetic inhomogeneities and I acknowledge M. Gasperini and G. Veneziano for valuable collaboration.

\section{References}

[1] G. D. Coughlan, W. Fischler, E. W. Kolb, S. Raby and G. G. Ross, Phys. Lett. B131, 59 (1983).

[2] A. S. Goncharov, A. D. Linde and M. I. Vysotsky, Phys. Lett. B147, 279, (1984).

[3] G. German and G. G. Ross, Phys. Lett. B172, 305 (1986).

[4] J. Ellis, N. C. Tsamish and M. Voloshin, Phys. Lett. B194, 291 (1987). 
[5] J. Ellis, D. V. Nanopoulos and M. Quiros, Phys. Lett B174, 291 (1986).

[6] A. Linde, Phys. Rev. D, 53 R4129 (1996).

[7] T. Banks, M. Berkooz and P. Steinhardt, Phys. Rev. D 52, 705 (1995).

[8] C. Lovelace, Phys. Lett. B135, 75 (1984);E. S. Fradkin and A. A. Tseytlin, Nucl. Phys. B261, 1 (1985); C. G. Callan et al., Nucl. Phys. B262, 593 (1985).

[9] E. Fischbach and C. Talmadge, Nature 356, 207 (1992).

[10] T. Damour and A. M. Polyakov, Nucl. Phys. B423, 532 (1994).

[11] M. Gasperini, Phys. Lett. B327, 214 (1994).

[12] G. Veneziano, Phys. Lett. B265, 287 (1991); M. Gasperini and G. Veneziano, Phys. Rev. D 50, 2519 (1994).

[13] D. H. Lyth and E. D. Stewart, Phys. Rev. Lett. 75, 201 (1995); Phys. Rev. D 53, 1784 (1996).

[14] E. M. Lifschitz, Zh. Eksp. Teor. Fiz. 16, 587 (1946); L. P. Grishchuk, Sov. Phys. JEPT 40, 409 (1975); A. A. Starobinsky, Pis'ma Zh. Eksp. Teor. Fiz. 30, 719 (1979).

[15] M. Gasperini, M. Giovannini and G. Veneziano, Phys. Rev. Lett. 75, 3796 (1995); Phys. Rev. D 52, 6651 (1992).

[16] L. Kofman, A. Linde and A. A. Starobinsky, Phys. Rev. Lett. 73, 3195 (1994); Phys. Rev. Lett. 76, 1011 (1996); E. W. Kolb, A. D. Linde and A. Riotto, Phys. Rev. Lett 77, 4290 (1996).

[17] J. Traschen and R. Brandenberger, Phys. Rev. D 42, 2491 (1990); Y. Shtanov, J. Traschen and R. Brandenberger, Phys. Rev. D 51, 5438 (1994).

[18] S.Yu. Khlebnikov and I.I. Tkachev Phys.Rev.Lett. 77, 219 (1996); Phys.Lett. B390, 80 (1997); PURD-TH-96-08, e-print Archive hep-ph/9610477 (unpublished). 
[19] M. Yoshimura, Prog. Theor. Phys. 94, 873 (1995); H. Fujisaki, K. Kumekawa, M. Yamaguchi and M. Yoshimura, Phys. Rev. D 53, 6805 (1996).

[20] D. Boyanovsky, H. J. De Vega, R. Holman and J. F. J. Salgado, Phys. Rev. D 54, 7570 (1996).

[21] D. Boyanovsky, D. Cormier, H. J. de Vega and R. Holman, Phys. Rev. D 55 (1997), in press.

[22] G. Veneziano, CERN-TH. 7502/94, Contribution to the PASCOS'94 Conference, Syracuse N.Y., (May 1994).

[23] D. Boyanovsky, H. de Vega, R. Holman, D.-S. Lee and A. Singh, Phys. Rev. D 51, 4419 (1995).

[24] M. Gasperini and M. Giovannini, Phys. Rev. D 47, 1519 (1993).

[25] L. D. Landau and E. Lifschitz, Mechanics page 80 ff. (Pergamon Press, Oxford, 1960).

[26] A. Erdelyi, W. Magnus, F. Obehettinger and F. R. Tricomi, Higher Trascendental Functions (Mc Graw-Hill, New York, 1953). 